\title{
Preserved Ratio Impaired Spirometry in a Spirometry Database
}

\author{
Andrei Schwartz, Nicholas Arnold, Becky Skinner, Jacob Simmering, Michael Eberlein, \\ Alejandro P Comellas, and Spyridon Fortis
}

\begin{abstract}
BACKGROUND: Spirometry results can yield a diagnosis of normal air flow, air flow obstruction, or preserved ratio impaired spirometry (PRISm), defined as a reduced FEV $_{1}$ or FVC in the setting of preserved $\mathrm{FEV}_{\mathbf{1}} / \mathrm{FVC}$. Previous studies have estimated the prevalence of PRISm to be 7-12\%. Our objective was to examine the prevalence of PRISm in a spirometry database and to identify factors associated with PRISm. METHODS: We performed a retrospective analysis of 21,870 spirometries; 1,616 were excluded because of missing data or extremes of age, height, or weight. We calculated the prevalence of PRISm in prebronchodilator and postbronchodilator pulmonary function tests. Subsequently, we calculated the prevalence of PRISm by various age, race, body mass index, and diagnosis categories, as well as by gender and smokers versus nonsmokers. Finally, in the subset of the cohort with $\mathrm{FEV}_{1}<$ lower limit of normal, we performed a multivariable logistic regression analysis to identify factors associated with PRISm. RESULTS: We identified 18,059 prebronchodilator spirometries, and $22.3 \%$ of these yielded a PRISm diagnosis. This prevalence remained stable in postbronchodilator spirometries (17.7\%), after excluding earlier pulmonary function tests for subjects with multiple pulmonary function tests $(20.7 \%$ in prebronchodilator and $24.3 \%$ in postbronchodilator), and when we limited the analysis to prebronchodilator spirometries that met American Thoracic Society criteria (20.6\%). The PRISm prevalence was higher in subjects 45-60 y old $(\mathbf{2 4 . 4 \% )}$ and in males $(23.7 \%)$ versus females $(17.9 \%)$. The prevalence rose with body mass index and was higher for those with a referral diagnosis of restrictive lung disease $\mathbf{5 0 \%}$ ). PRISm prevalence was similar between races and smokers versus nonsmokers. In a multivariable analysis, higher $\%$ of predicted $\mathrm{FEV}_{1}$ (odds ratio 1.51, 95\% CI 1.42-1.60), body mass index (odds ratio 1.52, 95\% CI 1.391.68), and restrictive lung disease (odds ratio 4.32 , 95\% CI $2.54-7.57$ ) were associated with a diagnosis of PRISm. Smoking was inversely associated (odds ratio $0.55,95 \%$ CI $0.46-0.65$ ) with PRISm. CONCLUSIONS: In a spirometry database at an academic medical center, the PRISm prevalence was $\mathbf{1 7 - 2 4 \%}$, which is higher than previously reported. Key words: preserved ratio impaired spirometry (PRISm); chronic obstructive pulmonary disease; lung volume measurements; plethysmography; respiratory function tests; spirometry. [Respir Care 2021;66(1):58-65. (C) 2021 Daedalus Enterprises]
\end{abstract}

\section{Introduction}

Abnormal nonobstructed spirometry, known as preserved ratio impaired spirometry (PRISm) or restrictive

\footnotetext{
Drs Schwartz and Arnold are affiliated with the Department of Internal Medicine, Division of General Internal Medicine, University of Iowa, Roy J. and Lucille A. Carver College of Medicine, Iowa City, Iowa. Drs Simmering, Eberlein, Comellas, and Fortis, and Ms Skinner, are affiliated with the Division of Pulmonary, Critical Care and Occupational Medicine, University of Iowa Hospital and Clinics, Iowa City, Iowa. Dr Fortis is affiliated with the Center for Access \& Delivery Research \& Evaluation (CADRE), Iowa City VA Health Care System, Iowa City, Iowa.
}

spirometry, occurs in $7-12 \%$ of the general population..$^{1-3}$ Among smokers in the general population with at least 10 pack-years of cigarette smoking who undergo spirometry after bronchodilator use, $12.5 \%$ have PRISm. ${ }^{4}$

\footnotetext{
The views expressed in this article are those of the authors and do not necessarily reflect the position or policy of the Department of Veterans Affairs or the United States Government.

Dr Fortis is supported by the Department of Veterans Affairs (Award \# 14380), the Comprehensive Access and Delivery Research and Evaluation Center (CIN 13-412), and has received grants from the American Thoracic Society, and Fisher \& Paykel. The other authors have disclosed no conflicts of interest.
} 
PRISm is associated with increased respiratory symptoms, respiratory exacerbations, and mortality. ${ }^{3-5}$ Unlike obstructive spirometry, PRISm does not indicate a specific lung disease. Subjects with PRISm represent a heterogeneous population with a wide range of lung function impairment and percent of radiographic emphysema. ${ }^{4}$ PRISm often requires further diagnostic investigation to identify the underlying lung disease.

Although the prevalence of PRISm has been studied in research cohorts in the general population and in smokers, the prevalence of PRISm in everyday clinical life is understudied. In a single-center study, $9.5 \%$ of all post-bronchodilator pulmonary function tests (PFTs) had $\mathrm{FEV}_{1}$ and FVC below the lower limit of normal while $\mathrm{FEV}_{1} / \mathrm{FVC}$, diffusing capacity for carbon monoxide, and total lung capacity (TLC) above the lower limit of normal. ${ }^{6}$ In our previous work, which included only PFTs that met the American Thoracic Society criteria for acceptability and repeatability, we reported that $37.4 \%$ of all pre-bronchodilator spirometries and 57\% of abnormal spirometries had PRISm. ${ }^{7}$

Smoking exposure, high body mass index, and reduced TLC have been associated with PRISm among a cohort of smokers without interstitial lung disease. ${ }^{8}$ The factors associated with PRISm in the clinical setting are not known. We hypothesized that the prevalence of PRISm in PFTs performed for clinical purposes is high. To investigate our hypothesis, we assessed the prevalence of PRISm in spirometries before and after bronchodilator use in our PFT labs that met the American Thoracic Society (ATS) criteria. We examined the association of PRISm with demographics, lung volume measurements, and referral diagnosis for the PFTs.

\section{Methods}

\section{Data Collection}

We conducted a retrospective analysis of data from PFTs performed at the University of Iowa Hospital from 1997 to 2018. The institutional review board approved the study protocol (201810837). After prebronchodilator spirometry, lung volumes were measured with plethysmography without bronchodilator. Postbronchodilator spirometry was performed 10 min after administration of 3 puffs of albuterol using a pressurized metered-dose inhaler and spacer. The PFT cohort in this is from the largest academic medical center in the state of Iowa and a referral center for interstitial lung disease, cancer, heart failure, and complex surgical and transplant patients.

Correspondence: Spyridon Fortis MD, UIHC Internal Medicine, 200 Hawkins Drive, C33 GH, Iowa City, IA 52242. Email: spyridon-fortis@uiowa.edu.

DOI: $10.4187 /$ respcare. 07712

\section{QUICK LOOK}

\section{Current knowledge}

Preserved ratio impaired spirometry (PRISm) is a spirometric pattern associated with respiratory symptoms and increased mortality. Individuals with PRISm represent a heterogenous group of diseases. It is possibly underdiagnosed and underappreciated. PRISm is likely more prevalent in patients in academic medical centers and in larger cohorts of medically complex patients.

\section{What this paper contributes to our knowledge}

PRISm was more prevalent in our spirometry database compared to previously reported prevalence in the general population. It remained very stable in several subanalysis groups. Individuals with PRISm represent a heterogenous population that cannot be fully explained by obesity, interstitial lung disease (or other restrictive lung disease) or differences in race.

We included subjects $>18$ y old. We excluded PFTs with no available reference values in NHANES III, ${ }^{9}$ including PFTs of patients from American Indian $(n=3)$, Asian $(n=164)$, or mixed race $(n=13)$; PFTs of patients $>80 \mathrm{y}$ old $(n=1,031)$; and PFTs of patients with height $<140$ $\mathrm{cm}(n=58),>217 \mathrm{~cm}(n=1)$, and with weight $<35 \mathrm{~kg}$ $(n=25)$. We collected PFT measurements as well as age, sex, height, race, and referral diagnosis from the PFT database in our PFT lab. Smoking status was retrieved from the electronic medical records.

Because the prevalence of PRISm may vary depending on whether pre- or postbronchodilator spirometry was used and whether the spirometry met the ATS criteria, we assessed PRISm prevalence in the following groups: (1) all prebronchodilator and postbronchodilator spirometries, (2) unique PFTs for each individual (if there were multiple PFT for a single individual, we kept the most recent), and (3) only prebronchodilator spirometries that met the ATS criteria.

\section{Definitions and Outcomes}

PRISm was defined as $\mathrm{FEV}_{1} / \mathrm{FVC} \geq$ lower limit of normal and $\mathrm{FEV}_{1}<$ lower limit of normal using reference values from the third National Health and Nutrition Examination Survey (NHANES III). ${ }^{9}$ Obstruction was defined as $\mathrm{FEV}_{1} / \mathrm{FVC}<$ lower limit of normal, and normal spirometry was defined as $\mathrm{FEV}_{1} / \mathrm{FVC} \geq$ lower limit of normal and $\mathrm{FEV}_{1} \geq$ lower limit of normal. Referral diagnoses were divided into 5 categories based on codes from the International Classification of Diseases, Tenth Revision: interstitial lung disease, restriction, obstructive lung diseases, other respiratory disease, miscellaneous, and none when 


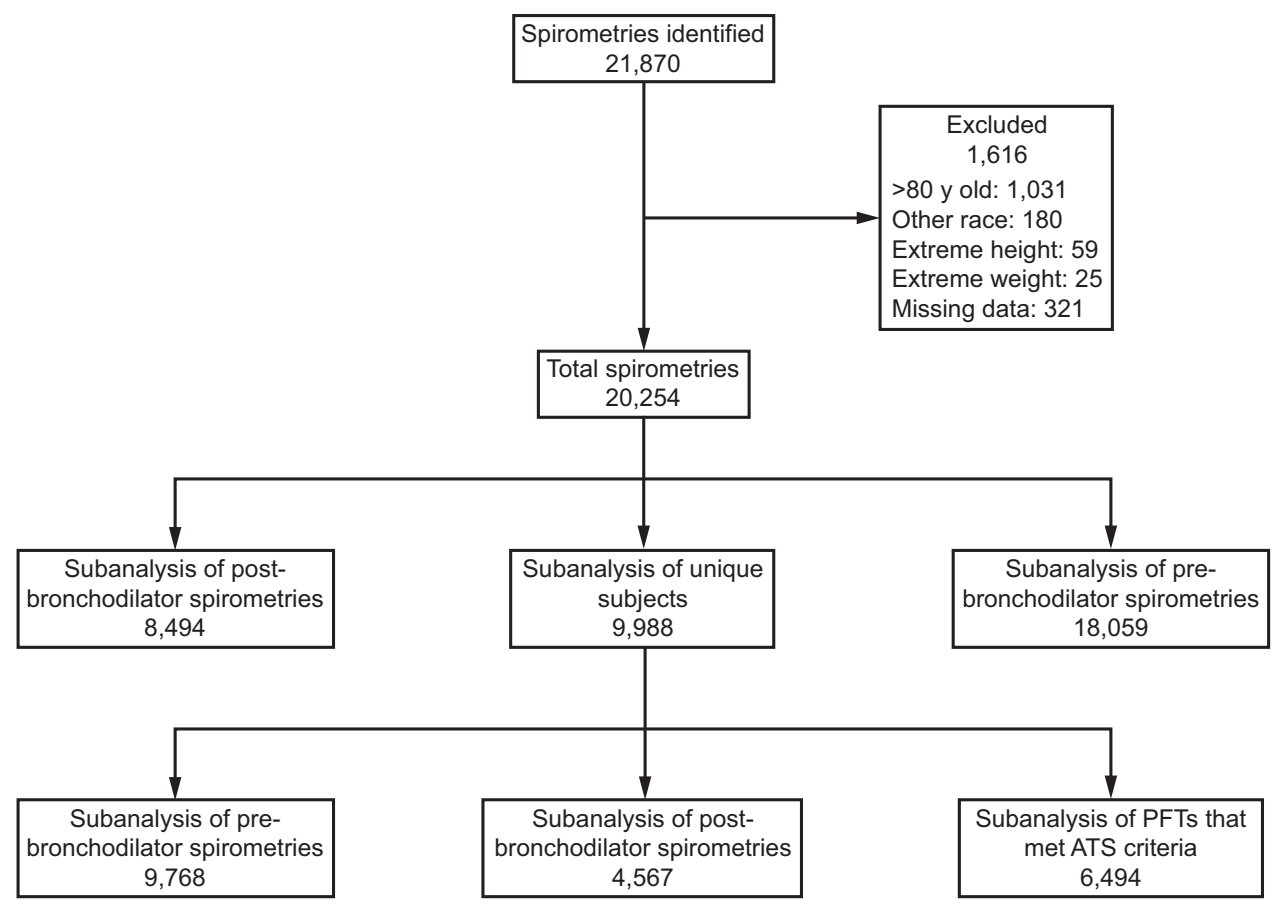

Fig. 1. Flow chart. PFT = pulmonary function tests; ATS = American Thoracic Society.

none was available. Other respiratory diseases were defined as those respiratory diseases or conditions that do not fall into the obstructive lung disease or interstitial lung disease category (eg, dyspnea, hypoxemia, cough, neuromuscular disease). No respiratory diseases or conditions were defined as miscellaneous. Lung volume was categorized into the following groups: (1) restriction defined as TLC $<$ lower limit of normal and RV/TLC $<$ upper limit of normal; (2) air trapping or hyperinflation defined as TLC $>$ upper limit of normal or RV/TLC > upper limit of normal; (3) unclassified, defined as TLC $<$ lower limit of normal and RV/TLC $>$ upper limit of normal; and (4) normal, defined as TLC $>$ lower limit of normal and TLC $<$ upper limit of normal combined with RV/TLC $<$ upper limit of normal. Severity of lung function impairment among PRISm was categorized into mild $\left(\mathrm{FEV}_{1} 50-80 \%\right.$ of predicted), moderate $\left(\mathrm{FEV}_{1} 30\right.$ $50 \%$ of predicted), and severe $\left(\mathrm{FEV}_{1}<30 \%\right.$ of predicted $)$.

\section{Statistical Analysis}

We stratified PFTs by age, race, sex, body mass index, smoking status, lung volume group, and diagnosis. We compared the prevalence of PRISm between groups using the Fisher exact test or the chi-square test when appropriate. We assessed concordance between spirometries before and after bronchodilator use to categorize spirometries as mormal, obstruction, or PRISm using $\kappa$ statistics. To identify factors associated with PRISm among patients with abnormal spirometry, we created a subset that included only patients with $\mathrm{FEV}_{1}<$ lower limit of normal (ie, normal spirometries were excluded). Univariate comparisons were then performed using the Fisher exact test for categorical variables and a Student $t$ test or Wilcoxon rank-sum test for normal and non-normal continuous variables, respectively, to identify variables associated with PRISm. Variables with a univariate $P<.05$ were considered candidates in the multivariable regression model. Variables were selected using a stepwise backward elimination to minimize the Akaike information criterion. ${ }^{10}$

In a sensitivity analysis, we calculated the prevalence of PRISm among spirometries that met the ATS criteria as prebronchodilator $\mathrm{FEV}_{1} / \mathrm{FVC} \geq$ lower limit of normal and FVC $\leq$ lower limit of normal. We used the R software package for all statistical analysis (http://www.r-project.org).

\section{Results}

We identified a total of 21,870 PFTs. We excluded 1,211 records of patients $>80 \mathrm{y}$ old or patients who were not white, African-American, or Hispanic. We also excluded 84 PFTs for extremes of height or weight, and 321 patients with no available spirometry (eg, PFT records included only lung volumes or the diffusing capacity for carbon monoxide). After these exclusions, a total of 20,254 records remained. Figure 1 shows the CONSORT diagram.

Of 20,254 PFTs that had at least a pre-bronchodilator or a post-bronchodilator spirometry, 18,059 records had prebronchodilator spirometries, and 8,494 records had postbronchodilator spirometries. The prevalence of PRISm was $22.3 \%(4,030)$ and $17.7 \%(1,507)$ in pre- 
Table 1. Baseline Characteristics of PFTs That Met American Thoracic Society Criteria

\begin{tabular}{lccc}
\hline \hline & PRISm & Normal & Obstruction \\
\hline Age & $55.61 \pm 15.11$ & $54.78 \pm 16.84$ & $56.69 \pm 15.47$ \\
Male & $722(53.80)$ & $1,263(41.00)$ & $1,054(5.90)$ \\
Height, $\mathrm{cm}$ & $1.7 \pm 0.11$ & $1.68 \pm 0.1$ & $1.7 \pm 0.1$ \\
Weight, $\mathrm{kg}$ & $96.75 \pm 30.45$ & $87.47 \pm 23.46$ & $85.62 \pm 26.25$ \\
Body mass index, $\mathrm{kg} / \mathrm{m}^{2}$ & $33.36 \pm 10.16$ & $30.81 \pm 7.86$ & $29.61 \pm 8.45$ \\
White & $1,265(94.30)$ & $2,849(92.40)$ & $1,944(93.90)$ \\
Active smoker & $710(52.90)$ & $1,354(43.90)$ & $1,354(65.40)$ \\
FEV before bronchodilator $\%$ & $59.44 \pm 13.23$ & $88.89 \pm 13.98$ & $57.42 \pm 20.5$ \\
FVC before bronchodilator, $\%$ & $64.2 \pm 14.46$ & $96.91 \pm 13.03$ & $85.43 \pm 30.29$ \\
Residual volume, $\%$ & $81.71 \pm 29.83$ & $82.83 \pm 28.97$ & $83.25 \pm 29.53$ \\
Total lung capacity, $\%$ & $83.35 \pm 18.24$ & & $81.72 \pm 17.12$ \\
Diagnosis category & & $339(11.00)$ & $137(6.60)$ \\
$\quad$ None & $148(11.00)$ & $491(15.90)$ & $917(44.30)$ \\
Obstructive & $211(15.70)$ & $704(23)$ & $269(13)$ \\
Restrictive & $270(20)$ & $1447(47.00)$ & $718(34.70)$ \\
Other respiratory symptoms & $583(43.40)$ & $101(3.30)$ & $29(1.40)$ \\
Other & $130(9.70)$ & &
\end{tabular}

Data are presented as mean $\pm \mathrm{SD}$ or $n(\%) . N=6,494$ subjects; PRISm: $n=1,342$ subjects; Normal: $n=3,082$ subjects; Obstruction: $n=2,070$ subjects.

$\mathrm{PFT}=$ pulmonary function test

PRISm $=$ preserved ratio impaired spirometry
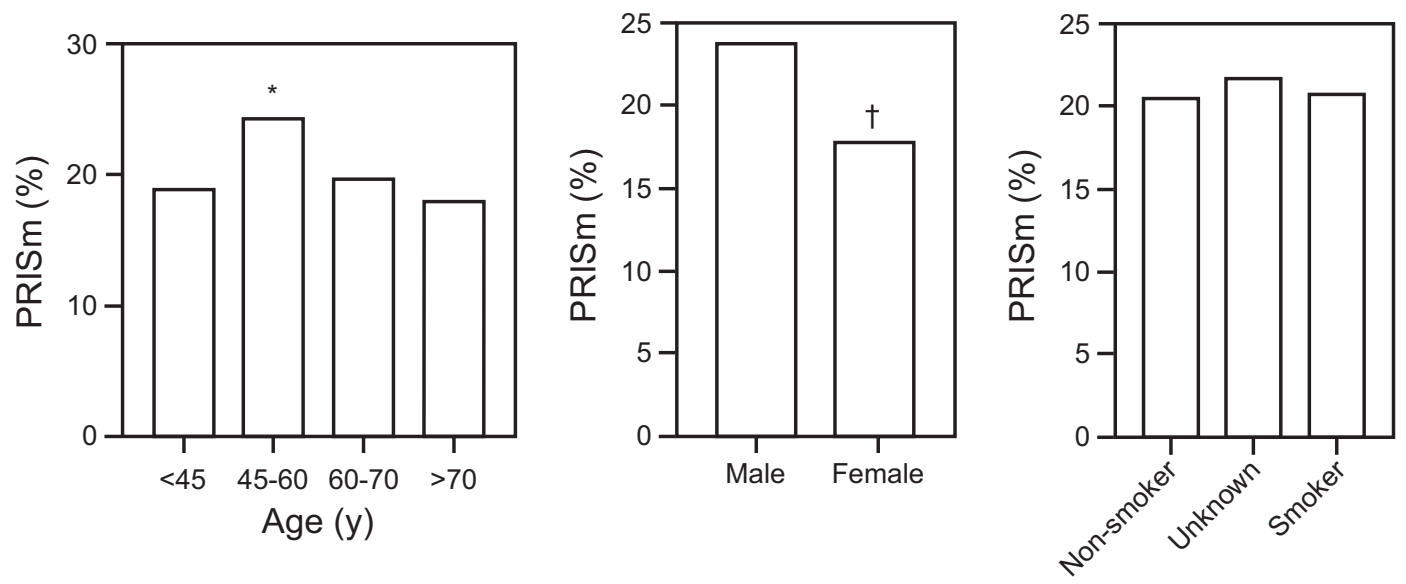

Fig. 2. Subgroup analysis of preserved ratio impaired spirometry (PRISm) based on age, sex, and smoking status. * $P<0.01$ between $45-60$ year old and all other age groups, ${ }^{\dagger} P<0.01$ between female and male sex.

and postbronchodilator spirometries, respectively. When we limited the analysis to unique subjects, we identified 9,988 records, with 9,768 prebronchodilator and 4,567 postbronchodilator spirometries. Of the 9,768 prebronchodilator spirometries, 2,031 $(20.7 \%)$ were PRISm. Of 4,567 postbronchodilator spirometries, $1,110(24.3 \%)$ yielded PRISm.

\section{Characteristics of Spirometries That Met ATS Criteria}

Among the spirometries that met ATS criteria $(6,494)$, the prevalence remained stable when we limited the analysis to unique records that met the ATS criteria in the prebronchodilator spirometries $(1,342$ out of $6,494,20.6 \%)$.

Table 1 shows the baseline characteristics of subjects who met ATS criteria. Figure 2 and Figure 3 show the prevalence of PRISm in several subgroups. The PRISm prevalence was higher in subjects 45-60 y old and in males versus females. The prevalence also rose with body mass index and with a referral diagnosis of restrictive lung disease $(50 \%)$. PRISm prevalence was similar between races (19.8\% in Hispanics, $20.9 \%$ in whites, $16.9 \%$ in AfricanAmericans) and smokers (20.8\%) versus nonsmokers $(20.5 \%)$. We then examined the severity of lung function 


\section{PRESERVEd RATIO IMPAIRED SPIROMETRY}
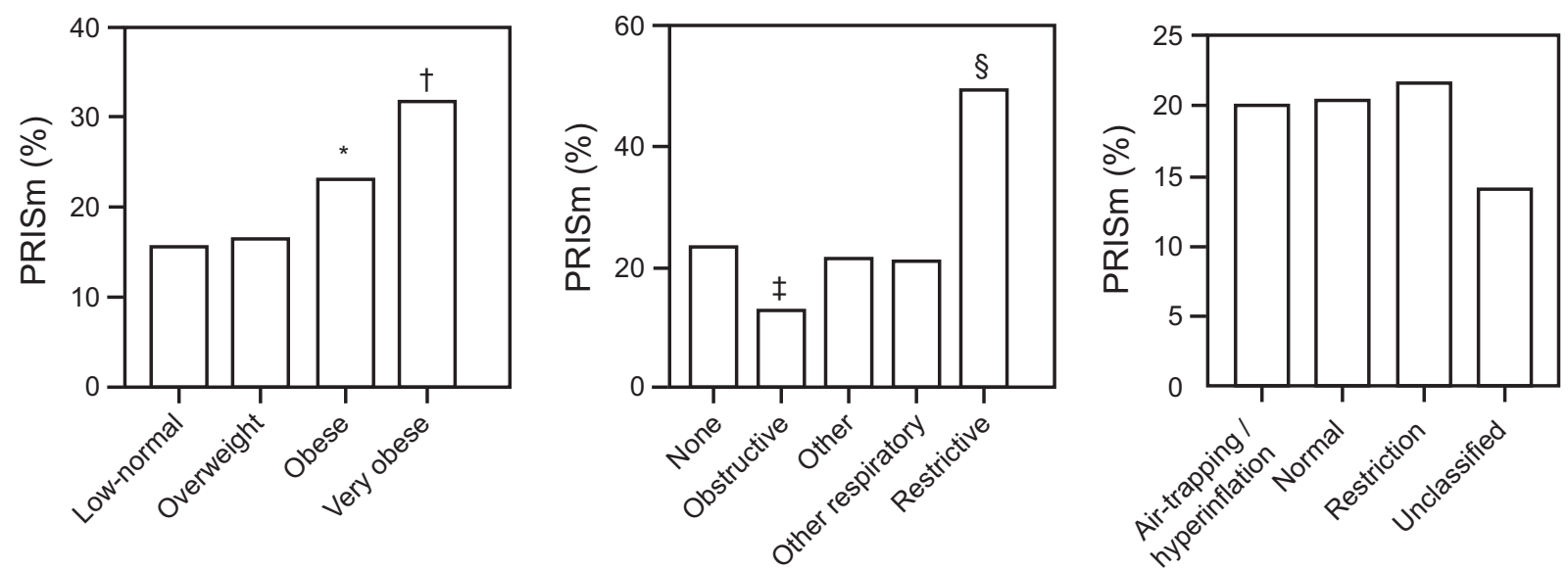

Fig. 3. Subgroup analysis of preserved ratio impaired spirometry (PRISm) based on body mass index, diagnosis category, and lung volumes. ${ }^{*} P<0.01$ between obese and low-normal/overweight, ${ }^{\dagger} P<0.01$ between very obese and all other weight groups, ${ }^{\ddagger} P<0.01$ between obstructive and none, ${ }^{\S} P<0.01$ between restrictive and all other PFT patterns.

Table 2. Agreement Between Pre- and Postbronchodilator Spirometry

\begin{tabular}{lcccr}
\hline \hline \multirow{2}{*}{ Prebronchodilator Spirometry } & \multicolumn{4}{c}{ Postbronchodilator Spirometry } \\
\cline { 2 - 5 } & Normal & Obstruction & PRISm & Total \\
\hline Normal & 746 & 99 & 307 & 1,152 \\
Obstruction & 220 & 884 & 101 & 1,205 \\
PRISm & 205 & 66 & 302 & 573 \\
Total & 1,171 & 1,049 & 710 & 2,930 \\
& & & & \\
$n=2,930$ subjects. $\kappa=0.125$ (ie, very poor agreement between before and after). & \\
PRISm $=$ preserved ratio impaired spirometry & & & \\
&
\end{tabular}

among PRISm based on $\mathrm{FEV}_{1}: 1,043(77.7 \%)$ had mild lung function impairment $\left(\mathrm{FEV}_{1} 50-80 \%\right), 261$ (19.4\%) had moderate lung function impairment $\left(\mathrm{FEV}_{1} 30-50 \%\right)$, and $38(2.8 \%)$ had severe lung function impairment $\left(\mathrm{FEV}_{1}\right.$ $<30 \%)$.

Using 2,930 records that had both pre- and postbronchodilator spirometries available, the agreement between preand postbronchodilator spirometry was poor with $\kappa=$ 0.125 (Table 2). The prevalence of PRISm was $19.6 \%$ and $23.2 \%$ in pre- and postbronchodilator spirometries, respectively. When we defined PRISm as prebronchodilator $\mathrm{FEV}_{1} / \mathrm{FVC} \geq$ lower limit of normal and $\mathrm{FVC} \leq$ lower limit of normal, the prevalence of PRISm was $22.3 \%$ (1,448 of 6,494).

Table 3 shows factors which were found to be associated with PRISm among those with abnormal spirometry $(n=$ 2,909). In multivariable analysis, higher $\mathrm{FEV}_{1}$ percent of predicted in $10 \%$ increments (odds ratio $1.51,95 \% \mathrm{CI}$ 1.42-1.60), body mass index in increments of $10 \mathrm{~kg} / \mathrm{m}^{2}$ (odds ratio 1.52, 95\% CI 1.39-1.68), and restrictive lung disease (odds ratio 4.32, 95\% CI 2.54-7.57) were associated with a diagnosis of PRISm. Smoking was inversely associated with PRISm (odds ratio 0.55 , 95\% CI $0.46-$ $0.65)$.

\section{Discussion}

Our results indicate that the prevalence of PRISm was $17-24 \%$ in a PFT lab dataset from an academic medical center. The prevalence remained relatively stable in several subanalysis groups, including a subanalysis of postbronchodilator spirometries after excluding earlier PFTs for subjects with multiple PFTs and a subanalysis that included only prebronchodilator spirometries that met ATS criteria. Among those with abnormal lung function, obesity and restrictive lung disease were associated with PRISm. Poor lung function (ie, lower $\mathrm{FEV}_{1}$ ) and smoking were inversely associated with PRISm.

Several studies assessed the prevalence of PRISm in the general population. In the NHANES I cohort, which included prebronchodilator spirometries from the general population, the prevalence of spirometries with FVC < $80 \%$ and $\mathrm{FEV}_{1} / \mathrm{FVC}>0.7$ was $9.2 \% .^{2}$ Using data from the ARIC study, Mannino et $\mathrm{al}^{3}$ noted that $7.1 \%$ of all subjects had prebronchodilator $\mathrm{FVC}<80 \%$ and a prebronchodilator $\mathrm{FEV}_{1} / \mathrm{FVC}>0.7$. Of abnormal spirometries, $16 \%$ had PRISm. In the Tucson epidemiological study, Guerra et al ${ }^{1}$ reported the PRISm prevalence to be $12 \%$. In that study, $46 \%$ of abnormal spirometries were PRISm. In the COPDGene cohort, which included only smokers and excluded patients with interstitial lung disease, the prevalence was $12.3 \% .^{5}$ In this study, we extended the literature by showing that the prevalence of PRISm was $17-24 \%$ in a PFT lab cohort at an academic medical center. The prevalence of PRISm was higher than in general-population studies. ${ }^{1-3}$ However, Jankowich et al $^{11}$ reported a prevalence of $19.9 \%$ in an African-American population in Mississippi, 
Table 3. Factors Associated With PRISm Among Individuals With Abnormal Spirometries

\begin{tabular}{|c|c|c|c|c|}
\hline & \multicolumn{2}{|c|}{ Univariable } & \multicolumn{2}{|c|}{ Multivariable } \\
\hline & Odds Ratio $(95 \% \mathrm{CI})$ & $P$ & Odds Ratio (95\% CI) & $P$ \\
\hline Age, per $10 \mathrm{y}$ & $0.92(0.88-0.97)$ & .03 & & \\
\hline Male & $1.04(0.90-1.21)$ & .001 & & \\
\hline Body mass index, per $10 \mathrm{~kg} / \mathrm{m}^{2}$ & $1.52(1.40-1.65)$ & $<.001$ & $1.53(1.39-1.68)$ & $<.001$ \\
\hline White & $0.88(0.60-1.29)$ & .51 & & \\
\hline Hispanic & $1.26(0.62-2.58)$ & .53 & & \\
\hline Prebronchodilator $\mathrm{FEV}_{1}$, every $10 \%$ & $1.57(1.49-1.66)$ & $<.001$ & $1.51(1.43-1.61)$ & $<.001$ \\
\hline TLC & $1.04(0.93-1.16)$ & .29 & & \\
\hline RV/TLC, every $10 \%$ & $0.92(0.79-1.07)$ & .55 & & \\
\hline Active smoking & $0.52(0.45-0.61)$ & $<.001$ & $0.55(0.46-0.65)$ & .045 \\
\hline \multicolumn{5}{|l|}{ Referral diagnosis } \\
\hline Obstructive & $0.20(1.17-1.95)$ & $<.001$ & $0.20(0.14-0.28)$ & $<.001$ \\
\hline Other & $0.95(0.69-1.30)$ & .76 & $0.89(0.63-1.26)$ & .52 \\
\hline Other respiratory symptoms & $0.71(0.54-0.94)$ & .02 & $0.71(0.52-0.96)$ & .03 \\
\hline Restrictive & $3.91(2.37-6.71)$ & $<.001$ & $4.32(2.54-7.57)$ & $<.001$ \\
\hline
\end{tabular}

which corroborated previous studies that reported higher PRISm in African-Americans. ${ }^{8,12}$

Prior reports have used variable definitions for PRISm, such as FVC $<80 \% \%^{1-3}$ or $\mathrm{FEV}_{1}<80 \%$ combined with an $\mathrm{FEV}_{1} / \mathrm{FVC} \geq 0.7 .^{4,13} \mathrm{FEV}_{1}$ and $\mathrm{FVC}$ are concomitantly reduced, whereas an isolated reduction in $\mathrm{FEV}_{1}$ or $\mathrm{FVC}$ is infrequent. ${ }^{7,14}$ Iyer et $\mathrm{al}^{14}$ reported that, among all abnormal nonobstructed spirometries, $85.5 \%$ had both reduced $\mathrm{FEV}_{1}$ and FVC, whereas $11.2 \%$ had only low $\mathrm{FEV}_{1}$, and $3.3 \%$ had isolated low FVC. Our previous work indicated that isolated reduction in $\mathrm{FEV}_{1}$ was only $4.7 \%$ of all abnormal nonobstructed spirometries. ${ }^{7}$ In this cohort, prevalence did not vary whether PRISm was defined as FVC $\leq$ lower limit of normal with $\mathrm{FEV}_{1} / \mathrm{FVC} \geq$ lower limit of normal, or as $\mathrm{FEV}_{1} \leq$ lower limit of normal with $\mathrm{FEV}_{1} / \mathrm{FVC} \geq$ lower limit of normal. Other studies used spirometry before bronchodilator use, ${ }^{1-3,11}$ and others used spirometry after bronchodilator use. ${ }^{4}$ Although the prevalence of PRISm in our study is similar regardless of whether spirometry was done before or after bronchodilator use, Table 2 shows their agreement being poor $(\kappa=0.125)$. Previous COPD studies have reported that pre-and postbronchodilator spirometries have similar predictive values based on correlation with clinical outcomes. ${ }^{10,15}$ Further research should assess the correlation of PRISm based on pre- and postbronchodilator spirometries with clinical outcome to assess whether postbronchodilator offers better results.

PRISm is often considered to be a result of obesity. The body mass index of individuals with PRISm is higher than that of smokers with normal spirometry or COPD. ${ }^{8}$ This is in agreement with our findings. Nevertheless, a landmark study by Jones and Nzekwu ${ }^{16}$ indicated that, although body mass index is inversely associated with FVC, obesity is unlikely to reduce FVC below the lower limit of normal in individuals with no respiratory disease. Among subjects undergoing preoperative evaluation for bariatric surgery with a body mass index at least of $35 \mathrm{~kg} / \mathrm{m}^{2}$, only $3 \%$ had an FVC $<$ lower limit of normal. ${ }^{17}$

Further, PRISm has been considered equivalent to restrictive respiratory disease. Although it is likely true that PRISm occurs in interstitial lung diseases, overall the prevalence of interstitial lung disease is very low, and it likely represents only a very small proportion of PRISm. Moreover, the prevalence of PRISm in the COPDGene cohort, which excluded interstitial lung disease, is 9$12.5 \%{ }^{4}$

If neither obesity nor interstitial lung disease are responsible for the larger proportion of PRISm, then what causes PRISm? Individuals with PRISm represent a heterogenous population. In smokers with PRISm, body mass index ranges from 17.2 to $53.8 \mathrm{~kg} / \mathrm{m}^{2}, \mathrm{FEV}_{1}$ ranges from $44 \%$ to $79 \%$, and radiographic emphysema ranges from $0.01 \%$ to $11.43 \% .^{1}$ A proportion of PRISm may be related to abnormal lung growth in early life. Seventeen percent of the general population had PRISm in their early 30s. ${ }^{18}$ Nevertheless, the most common respiratory diseases that can cause lung function impairment are obstructive lung diseases like asthma and COPD. It is very likely that obstructive lung diseases are responsible for most PRISm cases. Increased body mass index in combination with obstructive lung disease may result in abnormal spirometry with preserved $\mathrm{FEV}_{1} / \mathrm{FVC}$. O'Donnell and colleagues ${ }^{19}$ reported that obesity results in increased $\mathrm{FEV}_{1} / \mathrm{FVC}$ in subjects with COPD. In a recent study, Wijnant and colleagues ${ }^{13}$ noted that half of the PRISm subjects in the general population developed obstructive spirometry after a 


\section{Preserved RATIo IMPAIREd SPIROMETRY}

few years. In the Lovelace cohort, which included smokers from the general population ( $80 \%$ women), Sood et $\mathrm{al}^{20}$ reported that a proportion of subjects with COPD may have PRISm in follow-up spirometries and, vice versa, a proportion of PRISm individuals may have COPD. There is spirometric pattern change between COPD and PRISm over time. ${ }^{4,20}$ This variation occurs mostly in mild and moderate lung impairment, given that there are no PRISm individuals with $\mathrm{FEV}_{1}<44 \%$ in heavy smokers with no interstitial lung disease. ${ }^{8}$ In our cohort, increasing $\mathrm{FEV}_{1}$ percent of predicted was inversely associated with PRISm and confirms that.

Table 2 shows the poor agreement in our database based on whether pre- or postbronchodilator spirometry is used $(\kappa=0.125)$. Of 573 prebronchodilator PRISm, 205 returned to normal after bronchodilator use (likely an increase in both $\mathrm{FEV}_{1}$ and $\mathrm{FVC}$ ), suggesting a possible early obstructive (and partially reversible) test; 66 became obstructive, likely due to predominant bronchodilator response in FVC, which is common in COPD. ${ }^{21}$ These findings suggest that $47 \%$ (271 of 573) of these prebronchodilator PRISm may have early or mild obstructive disease that would only be revealed if these patients were given a bronchodilator. In the reciprocal analysis, of 710 postbronchodilator PRISm, 101 had prebronchodilator obstruction that normalized after bronchodilator use, indicating a larger increase in $\mathrm{FEV}_{1}$ compared to $\mathrm{FVC}$ and resulting in a normal $\mathrm{FEV}_{1} / \mathrm{FVC}$, which is typically seen in asthma (eg, flow response). Of 710 postbronchodilator PRISm, 307 had normal prebronchodilator spirometry. This might be explained by poor postbronchodilator effort, or it could be due to paradoxical bronchodilator response. ${ }^{22}$ The above results suggest that both pre- and postbronchodilator should be considered even when prebronchodilator test is not obstructive because administration of a bronchodilator might give more information on the pulmonary physiology.

Our study has several limitations. First, the use of retrospective data and using referral diagnosis by International Classification of Diseases codes might have biased the statistical analysis. Second, our spirometry data are spread over more than 20 years, from 1997 to 2018, with different spirometers and plethysmographs being used. Third, our study only looked at the prevalence of PRISm, and we have neither clinical follow-up data about the subjects nor occupational or environmental exposure history. Fourth, the PFT cohort in this study is from an academic medical center, which is different from a community hospital; our study sample is racially homogenous with $92.9 \%$ being white, which is a good representation of that proportion of the population of Iowa (90.1\% white according to the 2010 Census $^{23}$ ), but it may limit the generalizability of our study. Fifth, our PFT lab performed the postbronchodilator spirometry $10 \mathrm{~min}$ after 3 puffs of albuterol, which may have led to an underestimation of bronchodilator response.
These limitations do not undermine the strengths of our study, ie, the large sample size, the fact that all PFTs were performed in the same lab, and the reproducibility in several subgroup analysis. The fact that different spirometers and plethysmographs were used across 20 years increases our work's external validity.

\section{Conclusions}

PRISm is a spirometric pattern associated with respiratory symptoms and increased mortality. This study indicates that the prevalence of PRISm among subjects referred to a PFT lab in an academic medical center (17-24\%) is much higher than the prevalence in the general population. Although there is a discordance between pre- and postbronchodilator spirometry, the prevalence of PRISm when prebronchodilator spirometry was used is similar to the prevalence when postbronchodilator spirometry was used. Due to the poor agreement between spirometry before and after bronchodilator use, bronchodilators should be used to help distinguish between different pulmonary physiologies. Obesity and restrictive lung disease are associated with PRISm, while poor lung function and smoking are inversely associated with PRISm. Further research is needed to examine a diagnostic approach that will help identify the underlying diseases or conditions associated with PRISm and potential treatments.

\section{REFERENCES}

1. Guerra S, Sherrill DL, Venker C, Ceccato CM, Halonen M, Martinez FD, et al. Morbidity and mortality associated with the restrictive spirometric pattern: a longitudinal study. Thorax 2010;65(6):499-504.

2. Mannino DM, Buist AS, Petty TL, Enright PL, Redd SC. Lung function and mortality in the United States: data from the First National Health and Nutrition Examination Survey follow up study. Thorax 2003;58(5):388-393.

3. Mannino DM, Doherty DE, Buist AS. Global Initiative on Obstructive Lung Disease (GOLD) classification of lung disease and mortality: findings from the Atherosclerosis Risk in Communities (ARIC) study. Respir Med 2006;100(1):115-122.

4. Wan ES, Fortis S, Regan EA, Hokanson J, Han MK, Casaburi R, et al. Longitudinal phenotypes and mortality in preserved ratio impaired spirometry in the COPDGene study. Am J Respir Crit Care Med 2018;198(11):1397-1405.

5. Wan ES, Castaldi PJ, Cho MH, Hokanson JE, Regan EA, Make BJ, et al. Epidemiology, genetics, and subtyping of preserved ratio impaired spirometry (PRISm) in COPDGene. Respir Res 2014;15 (1):89.

6. Hyatt RE, Cowl CT, Bjoraker JA, Scanlon PD. Conditions associated with an abnormal nonspecific pattern of pulmonary function tests. Chest 2009;135(2):419-424.

7. Fortis S, Corazalla EO, Jacobs DR, Kim HJ. Persistent empiric COPD diagnosis and treatment after pulmonary function test showed no obstruction. Respir Care 2016;61(9):1192-1200.

8. Wan ES, Hokanson JE, Murphy JR, Regan EA, Make BJ, Lynch DA, et al. Clinical and radiographic predictors of GOLD-unclassified smokers in the COPDGene study. Am J Respir Crit Care Med 2011;184(1):57-63. 


\section{PRESERVEd RATIo IMPAIREd SPIROMETRY}

9. Hankinson JL, Odencrantz JR, Fedan KB. Spirometric reference values from a sample of the general US population. Am J Respir Crit Care Med 1999;159(1):179-187.

10. Fortis S, Eberlein M, Georgopoulos D, Comellas AP. Predictive value of prebronchodilator and postbronchodilator spirometry for COPD features and outcomes. BMJ Open Respir Res 2017;4(1):e000213.

11. Jankowich M, Elston B, Liu Q, Abbasi S, Wu W-C, Blackshear C, et al. Restrictive spirometry pattern, cardiac structure and function, and incident heart failure in African Americans. The Jackson Heart Study. Annals Am Thorac Soc 2018;15(10):1186-1196.

12. Mannino DM, Holguin F, Pavlin BI, Ferdinands JM. Risk factors for prevalence of and mortality related to restriction on spirometry: findings from the First National Health and Nutrition Examination Survey and follow-up. Int J Tuberc Lung Dis 2005;9(6):613-621.

13. Wijnant SRA, De Roos E, Kavousi M, Stricker BH, Terzikhan N, Lahousse L, et al. Trajectory and mortality of preserved ratio impaired spirometry: the Rotterdam Study. Eur Respir J 2020;55 (1):1901217.,

14. Iyer VN, Schroeder DR, Parker KO, Hyatt RE, Scanlon PD. The nonspecific pulmonary function test: longitudinal follow-up and outcomes. Chest 2011;139(4):878-886.

15. Mannino DM, Diaz-Guzman E, Buist S. Pre- and post-bronchodilator lung function as predictors of mortality in the Lung Health Study. Respir Res 2011;12:136.
16. Jones RL, Nzekwu MM. The effects of body mass index on lung volumes. Chest 2006;130(3):827-833.

17. Clavellina-Gaytan D, et al. Evaluation of spirometric testing as a routine preoperative assessment in patients undergoing bariatric surgery. Obes Surg 2015;25(3):530-536.

18. Lange P, Celli B, Agustí A, Boje Jensen G, Divo M, Faner R, et al. Lung-function trajectories leading to chronic obstructive pulmonary disease. N Engl J Med 2015;373(2):111-122.

19. O'Donnell DE, Deesomchok A, Lam Y, Guenette JA, Amornputtisathaporn $\mathrm{N}$, Forkert L, Webb KA. Effects of BMI on static lung volumes in patients with airway obstruction. Chest 2011;140(2):461-468.

20. Sood A, Petersen H, Qualls C, Meek PM, Vazquez-Guillamet R, Celli BR, et al. Spirometric variability in smokers: transitions in COPD diagnosis in a five-year longitudinal study. Respir Res 2016;17(1):147.

21. Fortis S, Comellas A, Make BJ, Hersh CP, Bodduluri S, Georgopoulos $\mathrm{D}$, et al. Combined forced expiratory volume in 1 second and forced vital capacity bronchodilator response, exacerbations, and mortality in chronic obstructive pulmonary disease. Ann Am Thorac Soc 2019;16 (7):826-835.

22. Bhatt SP, Wells JM, Kim V, Criner GJ, Hersh CP, Hardin M, et al. Radiological correlates and clinical implications of the paradoxical lung function response to $\beta 2$ agonists: an observational study. Lancet Respir Med 2014;2(11):911-918.

23. United States Census Bureau. 2010 Census. Available at: https://data. census.gov/cedsci. Accessed July 31, 2020. 